\title{
Correction to: Thoracic and cardiovascular surgery in Japan in 2016
}

\author{
Annual report by The Japanese Association for Thoracic Surgery
}

\begin{abstract}
Committee for Scientific Affairs, The Japanese Association for Thoracic Surgery ${ }^{1} \cdot$ Hideyuki Shimizu $^{2}$.

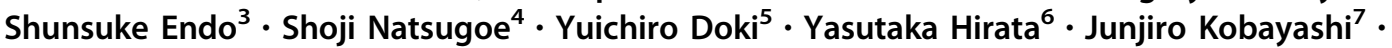
Noboru Motomura $^{8} \cdot$ Kiyoharu Nakano $^{9} \cdot$ Hiroshi Nishida $^{10} \cdot$ Morihito Okada $^{11} \cdot$ Yoshikatsu Saiki $^{12}$. Aya Saito ${ }^{8} \cdot$ Yukio Sato $^{13} \cdot$ Kazuo Tanemoto $^{14} \cdot$ Yasushi Toh $^{15} \cdot$ Hiroyuki Tsukihara $^{16} \cdot$ Shinji Wakui $^{17}$. Hiroyasu Yokomise ${ }^{18} \cdot$ Munetaka Masuda $^{19} \cdot$ Kohei Yokoi $^{20} \cdot$ Yutaka Okita $^{21}$
\end{abstract}

Published online: 24 April 2019

(C) The Author(s) 2019

\section{Correction to: General Thoracic and Cardiovascular Surgery https://doi.org/10.1007/s11748-019-01068-9}

In the original publication of the article, the values of the row "Norwood procedure", under "(3) Main procedure" in Table 3 were published incorrectly. The corrected part of the table is given in this Correction.

The original article can be found online at https://doi.org/10.1007/ s11748-019-01068-9.

Hideyuki Shimizu

survey-adm@umin.net

1 Committee for Scientific Affairs, The Japanese Association for Thoracic Surgery, Tokyo, Japan

2 Department of Cardiovascular Surgery, Keio University, 35, Shinanomachi, Shinjuku-ku, Tokyo, Japan

3 Department of Thoracic Surgery, Jichi Medical University, Shimotsuke, Japan

4 Department of Digestive Surgery and Breast and Thyroid Surgery, Kagoshima University, Kagoshima, Japan

5 Department of Gastroenterological Surgery, Osaka University Graduate School of Medicine, Suita, Japan

6 Department of Cardiac Surgery, The University of Tokyo Hospital, Tokyo, Japan

7 Department of Cardiovascular Surgery, National Cerebral and Cardiovascular Center, Suita, Japan

8 Department of Cardiovascular Surgery, Toho University, Sakura Medical Center, Sakura, Japan

9 Harajuku Rehabilitation Hospital, Tokyo, Japan

10 Rehabilitation, Tokyo Shinagawa Hospital, Tokyo, Japan
In addition, the last sentence in the first paragraph on page 14 should read as "The Norwood type I procedure was performed in 125 cases, with a relatively low hospital mortality rate of $14 \%$."
11 Department of Surgical Oncology, Hiroshima University, Higashihiroshima, Japan

12 Division of Cardiovascular Surgery, Tohoku University Graduate School of Medicine, Sendai, Japan

13 Department of Thoracic surgery, University of Tsukuba, Tsukuba, Japan

14 Department of Cardiovascular Surgery, Kawasaki Medical School, Kurashiki, Japan

15 Department of Gastroenterological Surgery, National Kyushu Cancer Center, Fukuoka, Japan

16 Department of Cardiothoracic Surgery, Graduate School of Medicine, The University of Tokyo, Tokyo, Japan

17 Cardiovascular Surgery, Nihon University Hospital, Tokyo, Japan

18 Department of General Thoracic Surgery, Faculty of Medicine, Kagawa University, Kida-gun, Kagawa, Japan

19 Department of Surgery, Yokohama City University, Yokohama, Japan

20 Department of Thoracic Surgery, Nagoya University Graduate School of Medicine, Nagoya, Japan

21 Cardio-aortic Center, Takatsuki General Hospital, Takatsuki, Japan 


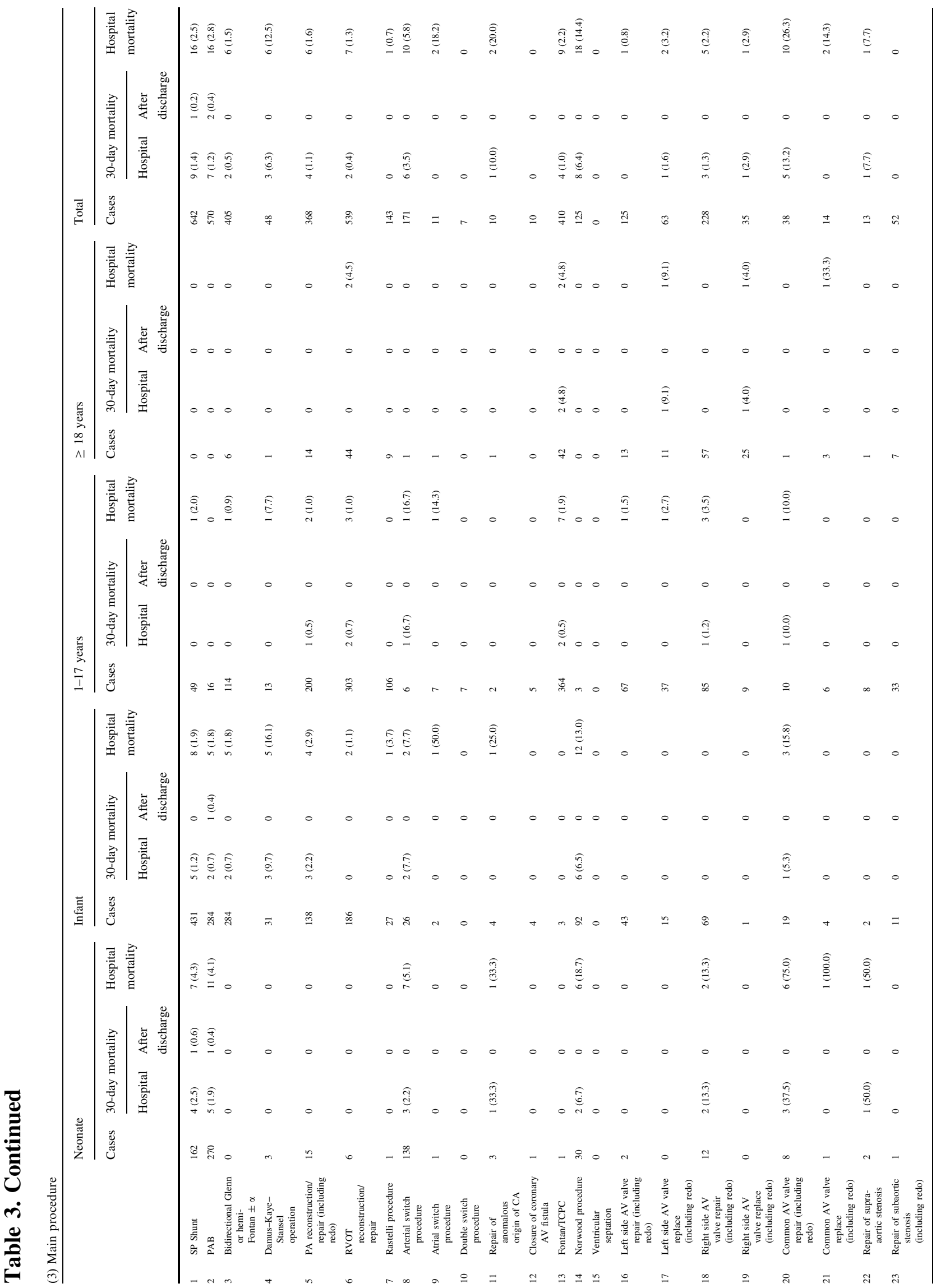




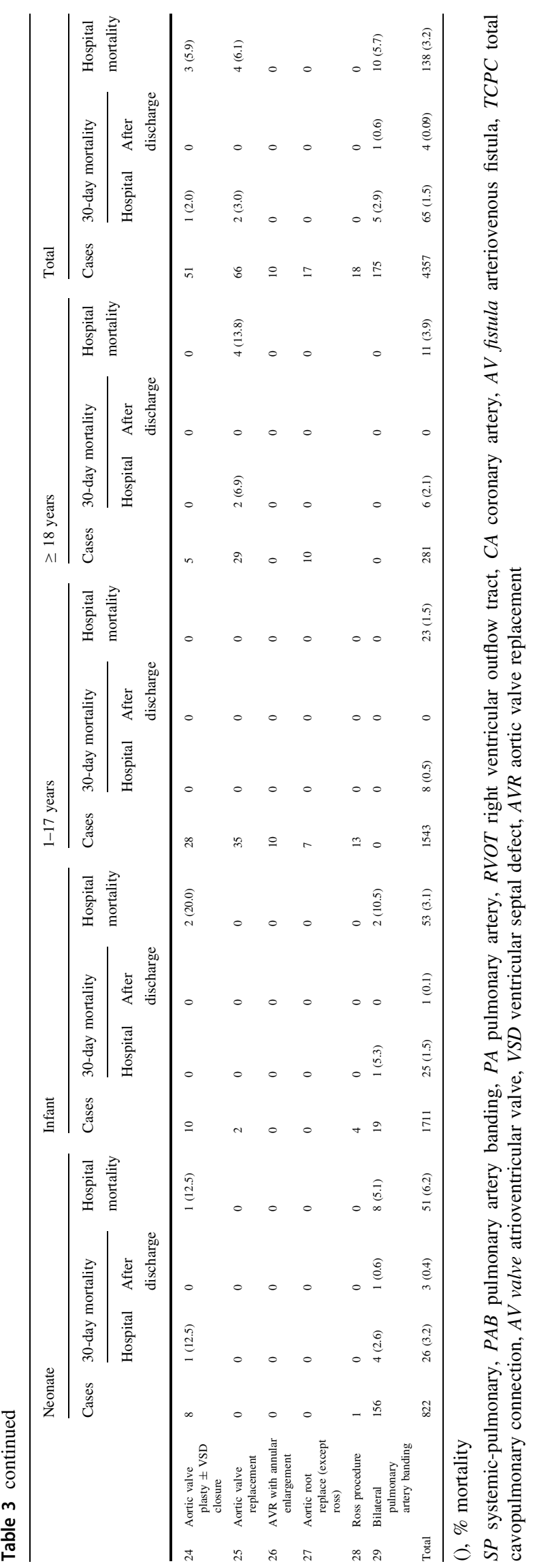

Open Access This article is distributed under the terms of the Creative Commons Attribution 4.0 International License (http://creative commons.org/licenses/by/4.0/), which permits unrestricted use, distribution, and reproduction in any medium, provided you give appropriate credit to the original author(s) and the source, provide a link to the Creative Commons license, and indicate if changes were made. 